\title{
Learning Historical and Cultural Contents via Mobile Treasure Hunting in Five-harbor District of Tainan, Taiwan
}

\author{
Stis $\mathrm{Wu}^{2}$, Alex Chang ${ }^{2}$, Maiga Chang ${ }^{3}$, Yu-Ren Yen ${ }^{4}$, Jia-Sheng $\mathrm{Heh}^{1}$ \\ ${ }^{1}$ Dept. of Information and Computer Engineering, Chung-Yuan Christian Univ., Taiwan \\ ${ }^{2}$ Dept. of Electronic Engineering, Chung-Yuan Christian Univ., Taiwan \\ ${ }^{3}$ School of Computing and Information Systems, Athabasca University, Canada \\ ${ }^{4}$ Department of Management Information System, Far East University, Taiwan \\ stiswu@gmail.com, aslada@mcsl.ice.cycu.edu.tw,maiga@ms2.hinet.net,yurenyen@gmail.com, \\ jsheh@ice.cycu.edu.tw
}

\begin{abstract}
Traditional formal education asks students studying in classroom and listening to teacher's lecturing. Recent years, teachers are taking students out from the classroom and doing field trip for learning history and cultural contents. Students can benefit from situated learning theory by interacting with the historical objects, local people, and cultural atmosphere. This research combines treasure hunting and mobile phone together to create a mobile treasure hunting learning situation for students learning historical and cultural contents during the field trip. In this mobile treasure hunting learning model, students act as hunters who can get hints through their mobile phones to find the treasures - the knowledge of historical and cultural contents. The mobile phones provide students not only display interface of treasure hunting maps and clues, but also position locator and treasure collector. In the experiment that eighteen elementary school students (5th Grade) learning in five-harbor district of Tainan, Taiwan.
\end{abstract}

Keywords: Treasure Hunting, Mobile Learning, Situated Learning, Mobile Phone, Elementary School, History Course, Culture Course, Field Trip

\section{Introduction \& Background}

Learning historical and cultural contents is better to bring the students to the historical site and make the students interact with the place and the objects [1]. Chang and Chang (2006) designed a treasure hunting learning model to stimulate students' learning motivation in outdoor activities and implemented the treasure hunting learning system [1]. This research extends the treasure hunting learning model that Chang and Chang proposed by combining the treasure hunting quests and built-in mobile phone functions.

Section 2 analyzes and designs the mobile treasure hunting learning model. Section 3 describes the experiment design and preliminary findings. Section 4 makes a conclusion and discusses the possible future works.

\section{Mobile Treasure Hunting Learning}

Figure 1 shows the architecture of the revised Treasure Hunting Learning Model. The architecture involves seven stages, basically teachers only get involved at stage 2 and 3, other stages involve only students. The first two stages in the architecture relate to students' learning and teachers' assessment. For example, the knowledge and concepts a student has learned can provide to the teacher via a paper-based exam or an online adaptive test (stage 1), the teacher can get idea about which topic the student might don't fully understand and which topic the student might have misconceptions on it and discover the concept the student might need to learn more in the field trip (stage 2). Before the student can start doing treasure hunting learning activity with his/her mobile phone (stage 4 to 7 ), the teacher has to build necessary stories first (stage 3).

Figure 2 shows how a treasure hunting wanted list is formed (stage 4). A wanted list is composed of the story and quest jigsaw edge pieces. The two adjacent edge pieces form many sub-goals. Each sub-goal contains background, description, guidance, and question elements as Figure 2 (right-hand side) and Figure 3 show. A student has to solve the sub-goals in 
sequence in order to get the treasure hunting clue for the next destination and to complete the wanted list puzzle.

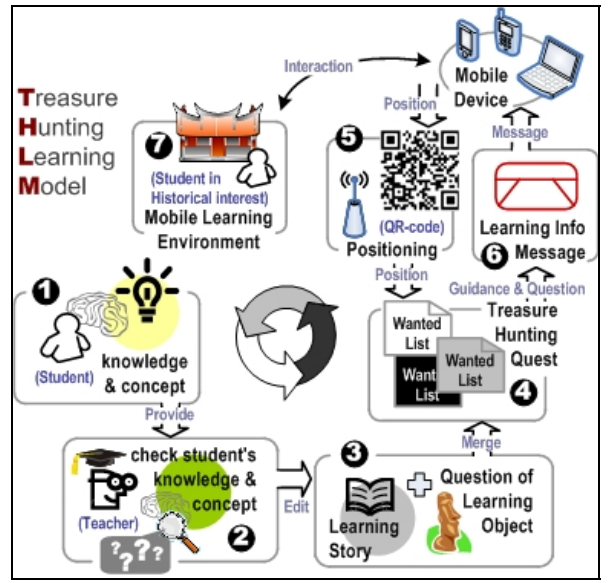

Figure 1. Architecture of Treasure Hunting Learning Model

Two parts, the background and the description, in the sub-goal of treasure hunting wanted list are associated with the story puzzle. For example, a background could be "When you go across the park, you can enter the Five-harbor District in history. Residents are sickening due to unknown disease and only you can rescue them."

Two parts, the guidance and the question, in the sub-goal of treasure hunting wanted list are associated with the quest puzzle. For examples, the guidance for leading students to go to specific destination will be "You need to locate Hai-An Temple and find the dragon pillar. After you found the dragon pillar, you can get the dragon's help by praying sincerely" and the question for asking students to learn will be "Please help the dragon to retrieve its power from the past by identifying the number of its claw fingers."

Students do treasure hunting learning activities with their mobile phones from stage 4 to stage 7 . At stage 4 , students can see different treasure hunting wanted list via their mobile phones. The students start their treasure hunting journey after pick-up a wanted list. Be noted, as mentioned earlier, the wanted lists are related to the topics students might don't fully understand and the topics students might have misconceptions on it.

The treasure hunting learning activity strongly requires student locations. The students can use their mobile phones with built-in camera to scan the QRcodes we put on different places in the five-harbor district in advance (stage 5).

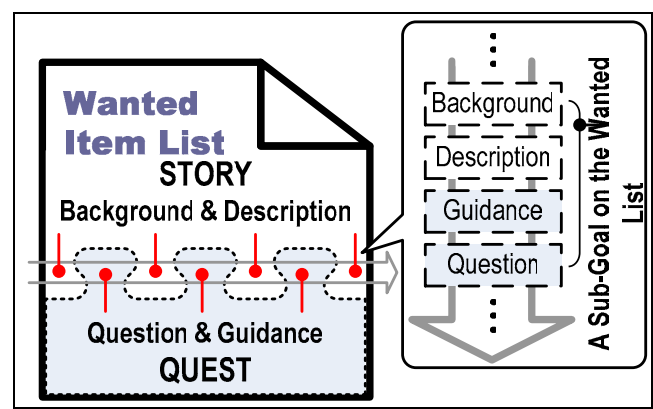

Figure 2. Stories and treasure hunting quests

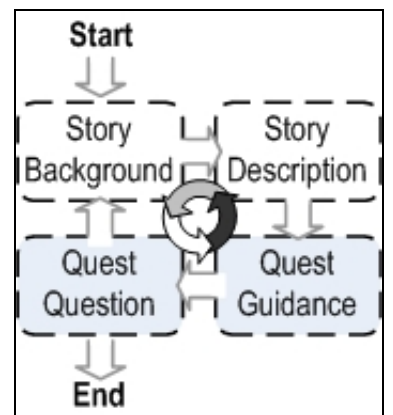

Figure 3. A sub-goal on the treasure hunting wanted list

The treasure hunting learning system can get the students' situations by interpreting the scanned QRcode and decide which information it should send to the students (stage 6). Stage 7 represents how students use their mobile phones to do the treasure hunting learning activities. The students use the built-in camera to scan QR-codes of historical sites and cultural objects to move around the learning areas; use short message to receive stories, quests, and questions of the treasure hunting wanted list; and, use touch screen to answer the questions.

\section{Experiment and Preliminary Findings}

This research did an experiment in Tainan Municipal Sie-jin Elementary School, Tainan, Taiwan. The experiment involved 5th grade eighteen students around 11 years old). Every student can have a mobile phone. The experiment asked the students doing treasure hunting learning activity to study history and culture of the Five-harbor District, Tainan, Taiwan. The Five-harbor District is an area which has plentiful cultural and historical relics. The treasure hunting learning trip takes two to three hours.

Before the students are allowed to start their treasure hunting trip, students have to search related information about the Five-harbor District on the Internet and to conduct slides with Microsoft PowerPoint delivering to the teacher (stage 1 in Figure 2). The teacher assesses students' slides to know what 
the students know already (stage 2 in Figure 2) and what treasure hunting wanted lists needed to prepared before the field trip starts (stage 3 in Figure 3).

The students use their mobile phones to do four tasks during their treasure hunting learning trip. The first task the students will do with their mobile phone is using the built-in camera to scan the QR-code attached on the artifacts and/or put beside the historical buildings to check if they are at correct location as Figure 4 shows. The second task the students will do is traveling to specific location and identifying specific object according to the message pop-up on the screen, for example, "You need to locate Hai-An Temple and find the dragon pillar".

After the students found the correct historical and cultural relics/artifact, the system sends relevant questions to the students. The third task the students have to do with their mobile phones is reading the questions from the phones and figuring the answers out by observing and discovering their surroundings as Figure 5 shows. After the students found the answers, the fourth task the students will do with their mobile phone is answering the questions by clicking the options or entering the short text.

When we designed the experiment, we had two hypotheses: (H1) the students like to use the treasure hunting learning system in their field trip; (H2) the students who like to do outdoor activity have higher motivation in doing treasure hunting learning activity. This research chooses the following two questions for the interviews. Beside the interviews, we also use questionnaires and video recordings to do qualitative data analysis.

1. Do you like to use treasure hunting learning system and mobile phone to learn history in the Five-harbor District?

2. Which one you like more? Doing learning activity at outside of classroom and home or doing learning activity in classroom and/or at home?

Almost all students have given very positive comments and feedbacks. Only two students have expressed fair comments to the revised treasure hunting learning model and activity. According the interview results and the behavior observations via video recording, we can say this revised treasure hunting learning model can work well for learning historical and cultural contents in field trip.

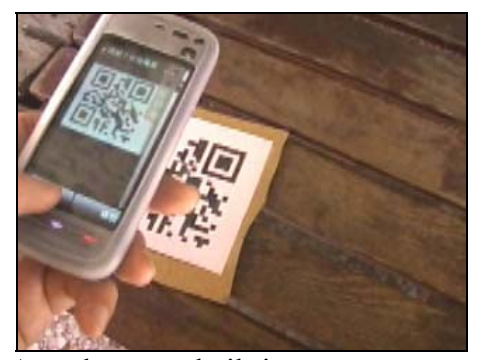

Figure 4. A student uses built-in camera to scan QR-code

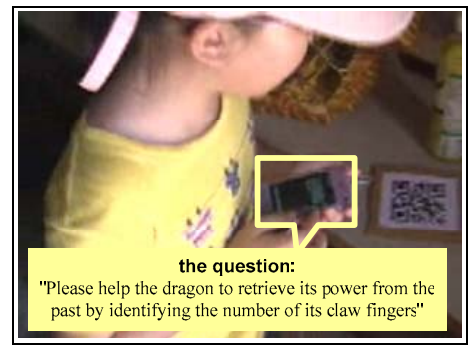

Figure 5. A student reads the question message

\section{Conclusions}

This research revises the treasure hunting learning model proposed by Chang and Chang in 2006 by using story and built-in functions that most of mobile phones may have today. We also do an experiment with eighteen 5th grade students participated in historical site, The Five-harbor District, Tainan, Taiwan. There are still some quantitative data analysis jobs should be done, e.g. gender difference and path analysis. Two research issues would be also considered to integrate into this research: context-awareness knowledge structure [3] and learning path planner [2].

\section{References}

[1] A. Chang, and M. Chang, "A Treasure Hunting Learning Model for Students Studying History and Culture in the Field with Cellphone", In the Proceedings of the 6th IEEE International Conference on Advanced Learning Technologies (ICALT 2006), July 2006, pp.106-108.

[2] R. Kuo, M.-C. Wu, A. Chang, M. Chang, and J.-S. Heh. Delivering Context-aware Learning Guidance in the Mobile Learning Environment based on Information Theory. In the Proceedings of the 7th IEEE International Conference on Advanced Learning Technologies, (ICALT 2007), Niigata, Japan, July 18-20, 2007, 362-366.

[3] S. Wu, A. Chang, M. Chang, T.-C. Liu, and J.-S. Heh. (2008). "Identifying Personalized Context-aware Knowledge Structure for Individual User in Ubiquitous Learning Environment", In the Proceedings of the 5th International Conference on Wireless, Mobile and Ubiquitous Technologies in Education, (WMUTE 2008), Beijing, China, March 23-26, 2008, pp. 95-99. (IEEE) 